\title{
Towards the Identification of Heat Induction in Chip Removing Processes via an Optimal Control Approach
}

\section{A Simplified Stationary Test Case for Drilling Processes}

\author{
Norman Lang · Jens Saak · Peter Benner \\ Steffen Ihlenfeldt · Steffen Nestmann · Klaus Schädlich
}

Received: date / Accepted: date

\begin{abstract}
This paper presents a linear-quadratic regulator (LQR) approach for solving inverse heat conduction problems (IHCPs) arising in production processes like chip removing or drilling. The inaccessibility of the processed area does not allow the measuring of the induced temperature. Hence the reconstruction of the heat source based on given measurements at accessible regions becomes necessary. Therefore, a short insight into the standard treatment of an IHCP and the related LQR design is provided. The main challenge in applying LQR control to the IHCP is to solve the differential Riccati equation (DRE). Here, a model order reduction approach is used in order to reduce the system dimension. The numerical results will show the accuracy of the approach for a problem based on data given by practical measurements.
\end{abstract}

Keywords inverse heat conduction - dynamical linear systems $\cdot$ optimal control $\cdot$ differential Riccati equation

\section{Introdution}

In many production fields, a certain heat load is induced during a machining process, as e.g., drilling or milling pro-

\footnotetext{
P. Benner ${ }^{1,2}$, N. Lang ${ }^{1, *}$ and J. Saak ${ }^{1,2}$

* Corresponding author

${ }^{1}$ Department of Mathematics, Technische Universität Chemnitz, Reichenhainer Straße 39/41, 09126 Chemnitz, Deutschland E-mail: benner/norman.lang/jens.saak@mathematik.tu-chemnitz.de

2 Max Planck Institute for Dynamics of Complex Technical Systems, Sandtorstraße 1, 39106 Magdeburg

E-mail: benner/saak@mpi-magdeburg.mpg.de
}

S. Ihlenfeldt, S. Nestmann and K. Schädlich

Fraunhofer IWU

Reichenhainer Straße 88, 09126 Chemnitz, Deutschland

E-mail: Steffen.Ihlenfeldt/

Steffen.Nestmann/Klaus.Schaedlich@iwu.fraunhofer.de cedures. These thermal loads affect the material properties and hence, cause deformations of the processed workpiece and the processing tool. Therefore, it is necessary to forecast the corresponding temperature field. However, in order to perform a simulation over the entire time horizon, the trajectories of the induced heat loads are required. Therefore, the modeling of chip removing processes and the associated determination of the actual induced amount of heat into the workpiece to be considered is a highly active research topic, see e.g., [20,24,25,26] and references therein. Since the chip removing process already requires a high accuracy model in order to identify the amount of heat entering the workpiece of interest, here we aim at reconstructing the heat inputs to the workpiece from observations of the temperature at accessible regions of the workpiece. The procedure investigated here in fact tries to avoid the sophisticated modeling of the entire chip removing process. Instead of performing a direct simulation of the heat creation and transfer process, we solve an inverse heat conduction problem (IHCP) [18] for the induced heat. Still the reconstruction requires a good knowledge of the heat transfer coefficients used in our boundary conditions and thus can heavily benefit from the investigation of the forward process.

The concrete setting of the application problem considered in this paper is described in Section 2. A short insight into the standard theory for solving the IHCP is given in Section 3.1. Exploiting the dynamical nature of the heat diffusion and at the same time avoiding complicated properties of the linear operator describing the heat propagation required in Section 3.1, we consider a linear quadratic regulator (LQR) approach to reconstruct the inaccessible heat loads induced by the machining process. This more control theoretic approach is described in Section 3.2. Section 4 states the corresponding solution strategy. Due to the intensive computational cost for derivation of the solution of the optimal control problem in the case of large-scale systems, 


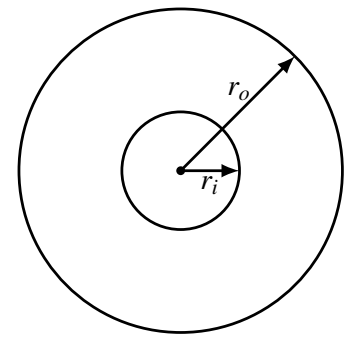

(a) Radii of hollow cylinder

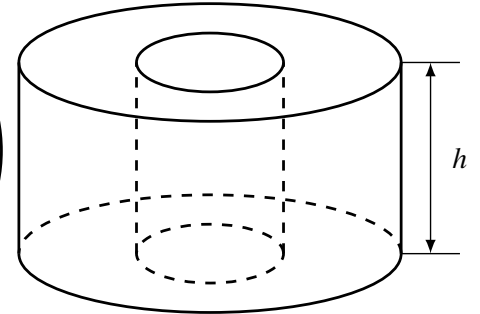

(b) Height of hollow cylinder
Figure 1: Model domain $\Omega$

in Section 5 we review a model order reduction (MOR) procedure. Finally, Section 6 presents an illustration of the MOR accelerated method and its realization for a simplified model example based on measured data. The problem under consideration aims at a drilling process for a certain workpiece as it is described in the following section.

\section{Problem setting}

We consider the 3-dimensional steel hollow cylinder given in Figure 1. The domain represents an experimental workpiece with an inner radius $r_{i}=47.75 \mathrm{~mm}$, an outer radius $r_{o}=113 \mathrm{~mm}$ and a height of $h=102 \mathrm{~mm}$. The measurements, we consider in the remainder are taken at the outer surfaces of the hollow cylinder (see Section 6). The corresponding partial differential equation describing the temperature field on $\Omega$ with Robin boundary conditions [27] on the boundaries $\Gamma_{k}$ reads

$$
\begin{aligned}
c_{p} \rho \partial_{t} \Theta & =\lambda \Delta \Theta \\
\lambda \partial_{v} \Theta & =\kappa_{k}\left(\Theta_{\text {ext }}-\Theta\right) \\
\lambda \partial_{\nu} \Theta & =0 \\
\Theta(0, .) & =\Theta_{0},
\end{aligned}
$$

$$
\begin{aligned}
& \text { in }(0, T) \times \Omega, \\
& \text { on }(0, T) \times \Gamma_{k}, k=1, \ldots, 3, \\
& \text { on }(0, T) \times \Gamma_{k}, k=4,
\end{aligned}
$$

where $\Gamma_{1}$ denotes the inner boundary, $\Gamma_{2}$ the outer one, $\Gamma_{3}$ the upper boundary and $\Gamma_{4}$ describes the bottom of the domain $\Omega$. The material parameters for the specific heat capacity, the density, the heat conductivity, and the heat transport coefficients for different boundaries $\Gamma_{k}$ are denoted by $c_{p}, \rho, \lambda, \kappa_{k}$, respectively. Here, we consider a steel workpiece with $c_{p}=500 \frac{\mathrm{m}^{2}}{\mathrm{~s}^{2} \mathrm{~K}}, \rho=7850 \frac{\mathrm{kg}}{\mathrm{m}^{3}}$ and $\lambda=46 \frac{\mathrm{kgm}}{\mathrm{s}^{3} \mathrm{~K}}$. Further, the problem was implemented with the heat transfer coefficients $\kappa_{2}=\kappa_{3}=50 \frac{\mathrm{W}}{\mathrm{m}^{2} \mathrm{~K}}$ and $\kappa_{1}=300 \frac{\mathrm{W}}{\mathrm{m}^{2} \mathrm{~K}}$.

Our goal is to find the induced temperature $\left.\Theta_{\text {ext }}\right|_{\Gamma_{1}}$ at the inner boundary $\Gamma_{1}$ from measurements $y$ at parts of the boundaries $\Gamma_{2}, \Gamma_{3}$ and $\Gamma_{4}$.

According to [14] an IHCP of the above structure is a severely ill-posed inverse problem since the solution $\left.\Theta_{\text {ext }}\right|_{\Gamma_{1}}$

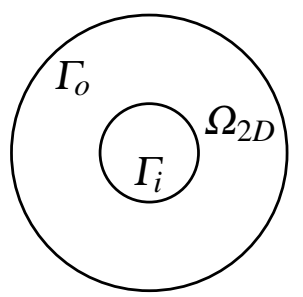

Figure 2: 2D hollow cylinder $\Omega_{2 D}$

of (1) does not depend continuously on the data $y$, i.e., already for slightly disturbed measurements the identified induced temperature may be quite different from the unperturbed.

\section{Inverse Problem versus Optimal Control}

\subsection{Standard solution technique for inverse problems}

The following statements are based on the results of [9]. Therein, equation (1) is considered on the two dimensional domain $\Omega_{2 D}$, depicted in Figure 2, with the inner boundary $\Gamma_{i}$ and the outer boundary denoted by $\Gamma_{o}$. For further simplification one considers a Dirichlet boundary value $g$ at the inner boundary $\Gamma_{i}$. This yields the forward formulation

$$
\begin{aligned}
c_{p} \rho \partial_{t} \Theta & =\lambda \Delta \Theta & & \text { in }(0, T) \times \Omega_{2 D}, \\
\lambda \partial_{\nu} \Theta & =q & & \text { on }(0, T) \times \Gamma_{o}, \\
\Theta & =g & & \text { on }(0, T) \times \Gamma_{i}, \\
(0, .) & =\Theta_{0} . & &
\end{aligned}
$$

of the heat conduction problem. Furthermore, it is assumed that the temperature denoted by $\hat{\Theta}$, as well as the heat flux $q$, are known on the outer boundary $\Gamma_{o}$ for each time instance $t \in[0, T]$. Since in addition the heat conductivity $\lambda$ is assumed to be constant, (2) can be formulated as

$\mathrm{K} g=\hat{\Theta}-\left.\Theta_{c}\right|_{(0, T) \times \Gamma_{o}}=: \tilde{\Theta}$,

which is linear in the quantity $g, \hat{\Theta}$ is the given temperature at the outer boundary and $\Theta_{c}$ denotes the solution of the constant part of equation (2) that can be computed as described in [9].

Finally, one ends up with a severely ill-posed operator equation $\mathrm{K} g=\tilde{\Theta}$ which can be solved for $g$ by any feasible method for this kind of problems. The severe ill-posedness leads to the necessity for regularization, which is often chosen to be of Thikonov type. In our approach a similar regularization is achieved by the choice of the weighting $R$ in the cost functional (6). The main problem with the approach in [9] is that the existence and the uniqueness of the solution are strongly related to conditions on the linear operator $\mathrm{K}$ which are hard to fulfill in practice. For details we refer 
to [9] and the references therein. Furthermore, the numerical treatment requires the discretization of (2) with respect to space and time at the same time. This leads to huge linear systems. Moreover, the flexibility of adapting the time discretization according to the features of $g$ is given up.

\subsection{Optimal control approach}

The time-dependence of the heat conduction problem naturally calls for a dynamical systems approach. In contrast to the a priori time discretization in the classical inverse problems setup, this allows us to use efficient time adaptive solution strategies, see Section 4. In addition, the standard theory of solving an IHCP crucially depends on rather complicated properties of the solution operator K. Therefore, we consider a control-theoretic approach [1,2] adapted to the framework of inverse problems, previously mentioned in [13]. There the searched for quantity is a heat flux input on a certain boundary part of the domain. The unknown heat flux input is reconstructed by given heat flux data taken at a an accessible part of the boundary. The main contribution of this paper is the treatment of the finite time horizon within the linear quadratic control problem described in [13]. First we reconsider the IHCP introduced in Section 1 defined on the domain $\Omega$ shown in Figure 1. In contrast to [13], here we consider the given data to be certain temperature measurements $\hat{\mathbf{y}}(t)$ on the boundaries $\Gamma_{2}$ and $\Gamma_{3}$. Since the external temperature $\Theta_{\text {ext }}$ at the inner boundary $\Gamma_{1}$ is the quantity of interest, and we want to apply an optimal control approach, we define $\left.\Theta_{\text {ext }}\right|_{\Gamma_{1}}=u$, i.e., we consider the heat source to be the control variable acting in the corresponding boundary condition at $\Gamma_{1}$. In order to obtain a finite dimensional dynamical system, a finite element (FE) discretization of equation (1) is considered and we end up with an inhomogeneous generalized state-space system of the form

$\mathbf{E} \dot{\mathbf{x}}=\mathbf{A x}+\mathbf{B u}+\mathbf{f}$.

Here, $\mathbf{E}$ is a FE mass matrix and $\mathbf{A}$ denotes the system matrix, consisting of the discrete Laplace operator and the boundary influences into the inside of $\Omega$ given by the boundary conditions. From here on, the state variable $\mathbf{x}(t)$ denotes the discretized version of the temperature field $\Theta$. The inhomogeneous part $\mathbf{f}$ consists of the external temperature introduced by the boundary conditions in equation (1), where $\lambda \partial_{\nu} \Theta=0$ on $\Gamma_{4}$, i.e., the bottom of $\Omega$ is insulated and therefore vanishes in $\mathbf{f}$.

The input matrix $\mathbf{B}$ describing the spatial influence of the induced heat to be determined, is the crucial ingredient making our approach feasible. We assume that at every given time $t$ we know where the heat source is located. For simplicity in this paper we expect this position to be fixed over time.
Defining an optimal control problem, the goal is to minimize the cost to drive the outputs $\mathbf{y}(t)$, defined by

$\mathbf{y}(t)=\mathbf{C x}(t)$,

with $\mathbf{C} \in \mathbb{R}^{p \times n}$ and $p$ being the number of measurements taken, to the given measurements $\hat{\mathbf{y}}(t)$ via the control $\mathbf{u}(t)$. That means, we aim at finding an (optimal) control $\mathbf{u}(t)$ such that the performance index

$$
\begin{aligned}
J(\mathbf{y}, \mathbf{u})= & \frac{1}{2} \int_{t_{0}}^{T}(\mathbf{y}(t)-\hat{\mathbf{y}}(t))^{T} \mathbf{Q}(\mathbf{y}(t)-\hat{\mathbf{y}}(t))+\mathbf{u}(t)^{T} \mathbf{R} \mathbf{u}(t) d t \\
& +\frac{1}{2}(\mathbf{y}(T)-\hat{\mathbf{y}}(T))^{T} \mathbf{S}(\mathbf{y}(T)-\hat{\mathbf{y}}(T))
\end{aligned}
$$

is minimized. The matrices $\mathbf{Q} \in \mathbb{R}^{p \times p}, \mathbf{R} \in \mathbb{R}^{m \times m}$ and $\mathbf{S} \in$ $\mathbb{R}^{p \times p}$ serve as weights to penalize large deviations in $\mathbf{y}-\hat{\mathbf{y}}$ or for regularization of (6). The non-integral term additionally penalizes deviations in the final state. Finally, the optimal control problem with the inhomogeneous generalized statespace constraints (4) is given by

$$
\begin{aligned}
\min \quad J(\mathbf{y}, \mathbf{u}) & \\
\text { subject to } \quad \mathbf{E} \dot{\mathbf{x}}(t) & =\mathbf{A x}(t)+\mathbf{B u}(t)+\mathbf{f}, \\
\mathbf{y}(t) & =\mathbf{C x}(t) .
\end{aligned}
$$

This is a so called tracking problem. The solution $\mathbf{u}$ of problem (7) is given (see, e.g. $[3,17]$ ) by the control law

$\mathbf{u}(t)=-\mathbf{R}^{-1} \mathbf{B}^{T}(\mathbf{X}(t) \mathbf{E x}(t)+\mathbf{w}(t))$,

where the time dependent matrix $\mathbf{X}(t)$ is the solution of the generalized differential Riccati equation (DRE)

$$
\begin{aligned}
\mathbf{E}^{T} \dot{\mathbf{X}}(t) \mathbf{E}=-\mathbf{C}^{T} \mathbf{Q C} & -\mathbf{A}^{T} \mathbf{X}(t) \mathbf{E}-\mathbf{E}^{T} \mathbf{X}(t) \mathbf{A} \\
& +\mathbf{E}^{T} \mathbf{X}(t) \mathbf{B R}^{-1} \mathbf{B}^{T} \mathbf{X}(t) \mathbf{E}
\end{aligned}
$$

with terminal condition $\mathbf{E}^{T} \mathbf{X}(T) \mathbf{E}=\mathbf{C}^{T} \mathbf{S C}$ and $\mathbf{w}(t)$ solves the adjoint state equation

$$
\begin{aligned}
\mathbf{E}^{T} \dot{\mathbf{w}}(t)= & -\left(\mathbf{A}-\mathbf{B} \mathbf{R}^{-1} \mathbf{B}^{T} \mathbf{X}(t) \mathbf{E}\right)^{T} \mathbf{w}(t) \\
& +\mathbf{C}^{T} \mathbf{Q} \hat{\mathbf{y}}(t)-\mathbf{X}(t) f,
\end{aligned}
$$

with terminal condition $\mathbf{E}^{T} \mathbf{w}(T)=-\mathbf{C}^{T} \mathbf{S} \hat{\mathbf{y}}(T)$. Both equations, (9) and (10), have to be solved backwards in time. It can be shown that one can easily switch between a forward and a backward solution ansatz and therefore use standard solution techniques. Note that in practice, the large-scale solution $\mathbf{X}(t) \in \mathbb{R}^{n \times n}$ is never formed explicitly, see [22]. We rather compute the feedback $\mathbf{K}(t)=-\mathbf{R}^{-1} \mathbf{B}^{T} \mathbf{X}(t) \mathbf{E} \in \mathbb{R}^{m \times n}$. Now, computing the adjoint state (10) and plugging Equation (8) into (4) we can compute the state $\mathbf{x}(t)$ and from that we obtain the desired input signal $\mathbf{u}(t)$. By construction $\mathbf{u}(t)$ then identifies the induced temperature we are searching for. 


\section{Solving the Optimal Control Problem}

In fact, the main computational bottleneck of solving the optimal control problem (7) is the solution of the matrix differential Riccati equation. Since the DRE is an ordinary differential equation (ODE), here one can think of any appropriate method to solve such. Still the DRE is matrix-valued. This leads to problems in terms of computational time and storage demands. In [7,8], the classes of backward differentiation formulas and Rosenbrock methods are adapted for use in the matrix-valued case. Here, we want to restrict ourselves to the Rosenbrock type methods (see e.g. [12] and the references therein).

Since we consider a linear time invariant (LTI) system, it is sufficient to treat the autonomous framework of Rosenbrock methods. A so called $s$-stage Rosenbrock scheme for autonomous ODE systems $\dot{x}=f(x)$ is defined as

$\mathbf{x}_{k+1}=\mathbf{x}_{k}+\tau \sum_{j=1}^{s} \beta_{j} \mathbf{k}_{j}^{(k)}$

$\mathbf{k}_{i}^{(k)}=f\left(\mathbf{x}_{k}+\tau \sum_{j=1}^{i-1} \alpha_{i, j} \mathbf{k}_{j}^{(k)}\right)+\tau \mathbf{J}_{k} \sum_{j=1}^{i} \gamma_{i, j} \mathbf{k}_{j}^{(k)}$,

$\forall i=1, \ldots, s$.

The quantities $\alpha_{i, j}, \gamma_{i, j}$ and $\beta_{j}$ denote the determining coefficients of the method, $\mathbf{J}_{k}$ is the Jacobian matrix $f^{\prime}\left(\mathbf{x}_{k}\right)$ and $\tau$ is the time step size. In every stage of the scheme a system of linear equations needs to be solved for the unknowns $\mathbf{k}_{i}$. To simplify representation and implementation, methods with $\gamma_{11}=\ldots=\gamma_{s s}=: \gamma$ are of special interest. The 1-stage Rosenbrock method (Ros1), e.g., is given as

$$
\mathbf{x}_{k+1}=\mathbf{x}_{k}+\tau \mathbf{k}_{1},
$$$$
\left(\mathbf{I}-\tau \mathbf{J}_{k}\right) \mathbf{k}_{1}=f\left(\mathbf{x}_{k}\right),
$$

with coefficients $\gamma=1, \beta_{1}=1$. It can easily be seen that this in fact is the linear implicit Euler method (see e.g., [12]). Following the statements in [7], the application of the Rosenbrock method (11) to the symmetric generalized DRE (9) leads to the solution of the generalized algebraic Lyapunov equation (GALE)

$$
\begin{aligned}
\overline{\mathbf{A}}_{k}^{T} \mathbf{X}_{k+1} \mathbf{E}+\mathbf{E}^{T} \mathbf{X}_{k+1} \overline{\mathbf{A}}_{k}= & -\mathbf{C}^{T} \mathbf{Q} \mathbf{C}-\frac{1}{\tau} \mathbf{E}^{T} \mathbf{X}_{k} \mathbf{E} \\
& -\mathbf{E}^{T} \mathbf{X}_{k} \mathbf{B} \mathbf{R}^{-1} \mathbf{B}^{T} \mathbf{X}_{k} \mathbf{E},
\end{aligned}
$$

with $\overline{\mathbf{A}}_{k}:=\mathbf{A}-\mathbf{B} \mathbf{R}^{-1} \mathbf{B}^{T} \mathbf{X}_{k} \mathbf{E}-\frac{1}{2 \tau} \mathbf{E}$. That means, we need to solve one GALE at every time step of the Ros 1 method to obtain the solution of the DRE. Increasing the order of the Rosenbrock method would linearly increase the number of ALEs one has to solve in each time step. Therefore, we restrict ourselves to the first order method described above. Again, the main effort to solve the DRE by a first order
Rosenbrock method is the solution of the GALE (12) in every step. Hence, it becomes necessary to use an efficient algorithm to achieve that. In the context of the optimal control problem (7), the solution of (12) usually is of low numerical rank. In $[6,16,21]$ it is shown that ALEs can be solved in an efficient way via a low-rank version of the alternating direction implicit (ADI) method and its extensions (see [4,5] for the most recent formulation). Note that when using higher order time integration methods, we recommend to use the ideas proposed in [15].

The model, describing the dynamical behavior of the temperature field induced by the input signal is of dimension $n=11318$. In combination with the time interval $t \in$ $[0,55320] s$ on which the measurements are taken (see Section 6), the available codes for the low-rank computations are not capable of solving the large-scale DRE regarding the computation times and especially storage demands. Therefore, we employ model order reduction (MOR) to reduce the system dimension and thus the computational complexity and the storage requirements for the solution.

\section{Model order reduction}

MOR allows us to find a system of much lower dimension approximating the full order model (FOM) and therefore the associated computations can be performed in a fraction of time. Further, the goal of any MOR method is to find a reduced order model (ROM)

$$
\begin{aligned}
\mathbf{E}_{r} \dot{\mathbf{x}}_{r} & =\mathbf{A}_{r} \mathbf{x}_{r}+\mathbf{B}_{r} \mathbf{u}+\mathbf{f}_{r}, \\
\mathbf{y}_{r} & =\mathbf{C}_{r} \mathbf{x}_{r}
\end{aligned}
$$

with $\mathbf{E}_{r}, \mathbf{A}_{r} \in \mathbb{R}^{r \times r}, \mathbf{B}_{r} \in \mathbb{R}^{r \times m}, \mathbf{C}_{r} \in \mathbb{R}^{q \times r}, \mathbf{f}_{r} \in \mathbb{R}^{r}$ and $r \ll n$, that approximates the original model (4) in such a way that the system behavior is well captured. That means, using the same input signal $\mathbf{u}$ as for the FOM, the output $\mathbf{y}_{r}$ of the $\mathrm{ROM}$ satisfies $\mathbf{y}_{r} \approx \mathbf{y}$. Therefore, the important states need to be determined with respect to a certain measure. In order to determine the ROM via a projection based MOR approach, we need to compute a pair of matrices $\mathbf{V}, \mathbf{W} \in \mathbb{R}^{n \times r}$ such that the reduced order system matrices are given in the form
$\mathbf{E}_{r}=\mathbf{W}^{T} \mathbf{E V}$,
$\mathbf{A}_{r}=\mathbf{W}^{T} \mathbf{A V}$,
$\mathbf{B}_{r}=\mathbf{W}^{T} \mathbf{B}$,
$\mathbf{C}_{r}=\mathbf{C V}$,
$\mathbf{f}_{r}=\mathbf{W}^{T} \mathbf{f}$.

In this contribution we consider the Balanced Truncation (BT) model order reduction approach. The BT method [19] ensures the stability of the ROM if the original model is stable and in addition an error bound for the reduction error is given [10]. The BT method keeps those state-space variables associated to a dominant energetic behavior. That means, the states, which are easy to steer and at the same 
time easy to observe are the states of interest. The measures for the controllability and observability of the system states are the so called system Gramians $\mathbf{P}$ and $\mathbf{Q}$, which can be computed as the solutions of the corresponding controllability and observability ALEs

$$
\begin{aligned}
\mathbf{A P E} \mathbf{E}^{T}+\mathbf{E P A}^{T} & =-\mathbf{B} \mathbf{B}^{T}, \\
\mathbf{A}^{T} \mathbf{Q E}+\mathbf{E}^{T} \mathbf{Q A} & =-\mathbf{C C}^{T} .
\end{aligned}
$$

As stated in Section 4, the large-scale Lyapunov Equations (14) and (15) can be solved efficiently using the low-rank ADI method. Given the solutions in the low-rank form $\mathbf{P} \approx \mathbf{Z}_{c} \mathbf{Z}_{c}^{T}$, $\mathbf{Q} \approx \mathbf{Z}_{o} \mathbf{Z}_{o}^{T}$, with $\mathbf{Z}_{c} \in \mathbb{R}^{n \times k_{c}}, \mathbf{Z}_{o} \in \mathbb{R}^{n \times k_{o}}$ and $k_{c}, k_{o} \ll n$, the projection matrices $\mathbf{V}, \mathbf{W}$ can be computed using the lowrank square root method (LRSRM) [23].

Note that the application of the MOR procedure introduces additional computational cost in terms of solving two extra Lyapunov equations in order to obtain the Gramians $\mathbf{P}, \mathbf{Q}$ of the system and the computation of the projection matrices $\mathbf{V}, \mathbf{W}$ via the LRSRM. Still, this is done once in advance of the solution of the DRE (9) and the entire forward solution of (4) using the feedback law (8). That is, the overall computational cost consists of solving two large Lyapunov equations for the MOR approach and the solution of the DRE (9) based on the reduced order matrices compared to the solution of one large-scale Lyapunov equation in every time step of the Ros1 method for the original model. At the same time the error in the solution at most increases by the controllable BT MOR error. That means, if the MOR error is kept below the discretization error we have present in the FE model anyway, then we can expect the solution to be as good as without the MOR.

\section{Numerical Experiments and Experimental Verification}

We consider a set of measurements taken at the accessible boundaries of the domain $\Omega$ presented in Figure 1 in Section 2. As mentioned in the introduction, the actual considered drilling process has been simplified. As a first test, the heat source representing the induced heat of the machining process is modeled by the application of a set of heating mats at the inner boundary $\Gamma_{1}$ as depicted in Figure 3. These heating elements are steered to a maximum temperature of around $50{ }^{\circ} \mathrm{C}$. The maximum is, compared to the slowly evolving temperature field inside the model domain $\Omega$, basically reached instantaneously. The measurements, necessary for the reconstruction approach, are taken by 6 sensors, equidistantly distributed at the insulated bottom (boundary $\Gamma_{4}$ ) of the hollow cylinder as presented in Figure 4 . The measurements are taken every $10 \mathrm{~s}$ over the time interval $t \in[0,55320] s$. As an example, the given data in Sensors 1,4 and 6 are presented in Figure 5. For the reconstruction of

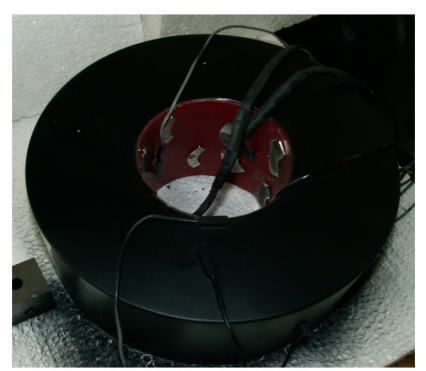

Figure 3: Heating mats at the inner boundary $\Gamma_{1}$ attached by magnets.

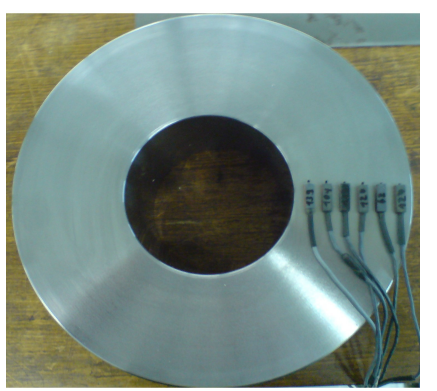

Figure 4: Sensors at the bottom boundary $\Gamma_{4}$ of the steel hollow cylinder.

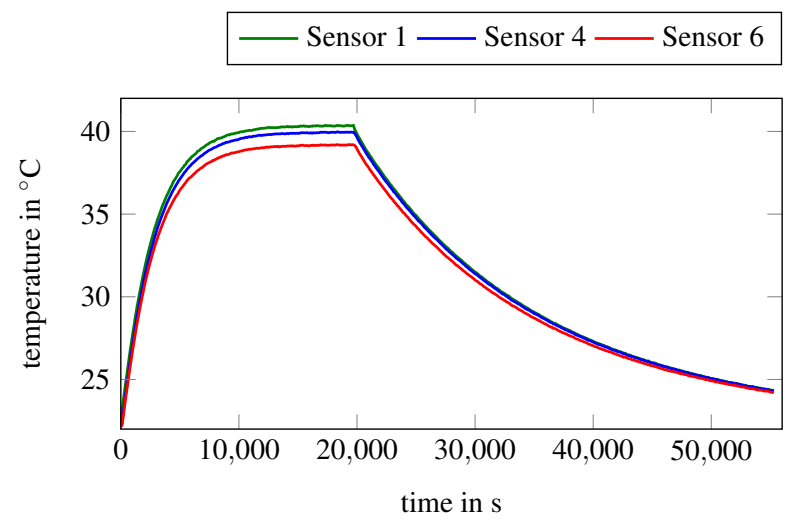

Figure 5: Measured temperature $\hat{\mathbf{y}}$ at sensor positions 1, 4, 6 .

the temperature input, induced by the heat mats, we consider the FE model of dimension $n=11318$ described already in the previous sections. The weighting matrices $\mathbf{Q}, \mathbf{R}, \mathbf{S}$ in the performance index (6) for simplicity are all chosen to be multiples of the identity of appropriate size. Note, the choice of the weightings can be further improved by using more advanced information of e.g., a weighted importance of the measurement nodes. Here, $\mathbf{Q}=2500 \cdot \mathbf{I}_{q}, \mathbf{R}=\mathbf{I}_{m}$ and $\mathbf{S}=100 \cdot I_{q}$. The scaling factors of the several identities are empirically determined in order to ensure a moderate deviation between the computed and the desired output (measurements). Considering the FE model based on the resolution of 


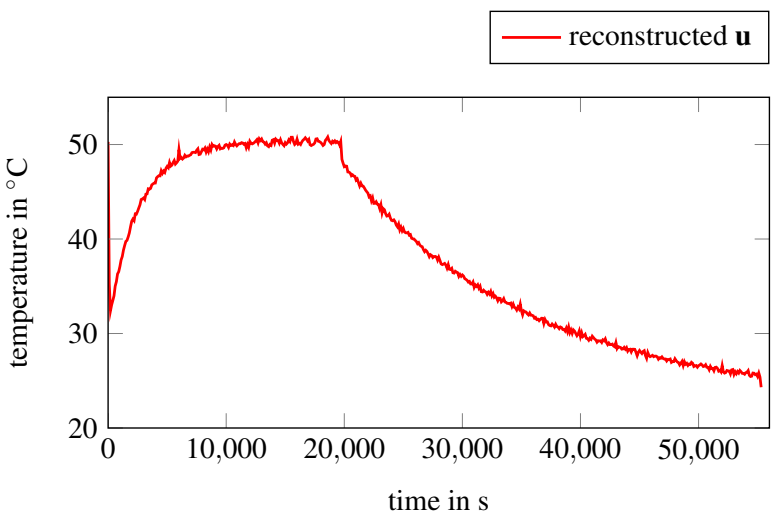

Figure 6: Reconstructed temperature input $\mathbf{u}$.

the utilized FE mesh we already introduced a certain modeling error strongly related to the maximum grid width and the FEs used, [11]. Controlling the BT error via the error bound we ensure the FE error to be dominant with respect to the MOR error. That means, the MOR procedure does not introduce a significant additional error. The ROM we could obtain by the BT procedure is of dimension $r=25$. That is, the DRE to be solved becomes a matrix-valued differential equation of dimension $r=25$ instead of $n=11318$. Further, remember that the reduced order model is constructed to approximate the outputs of the original model for the same given input signal $\mathbf{u}$. That means, the input reconstructed by the ROM is already the searched for quantity.

In order to interpret the results, the sensors are numbered with 1-6 from the inner to the outer boundary. Computing the solution of (7), we obtain the reconstructed temperature u presented in Figure 6. Since we cannot compare the result to a reference solution, we need to compare the measurements $\hat{\mathbf{y}}$ with the computed outputs $\mathbf{y}_{r}$ induced by the input $\mathbf{u}$. The results are depicted in Figure 7 and the corresponding relative error is given in Figure 8. We assume the heat model (4) to approximate the real process accurately enough, i.e., we have given, e.g., the correct material parameters. Then the result for the reconstructed temperature input $\mathbf{u}$ is quite reliable since the achieved errors for the outputs lie in the per mill range. The oscillations in $\mathbf{u}$ arise from the noise hidden in the measurements. These uncertainties can be reduced by filtering the given measurements or using a linear quadratic Gaussian (LQG) design [17] instead of the LQR approach described here. Still, the latter will lead to the solution of an additional, so called, filter differential Riccati equation. The application of the LQG design and its benefits will be investigated in the future.

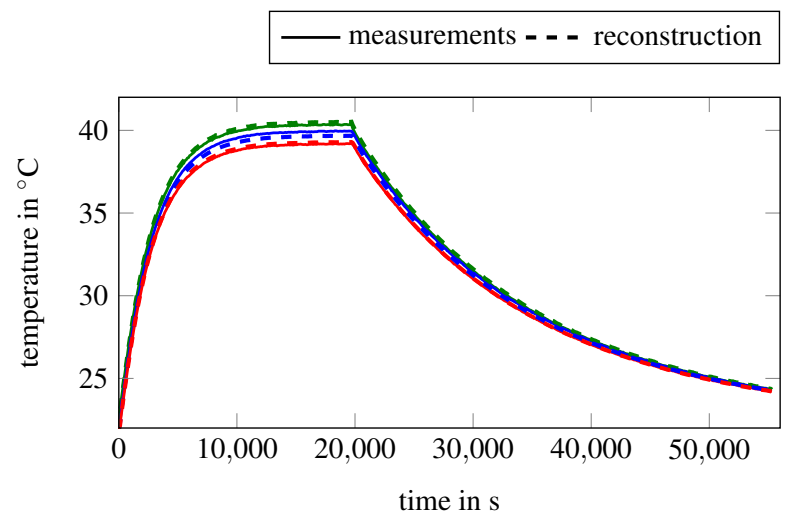

Figure 7: Comparison of the measured outputs $\hat{\mathbf{y}}$ and computed outputs $\mathbf{y}_{r}$ for Sensors 1,4 and 6 .

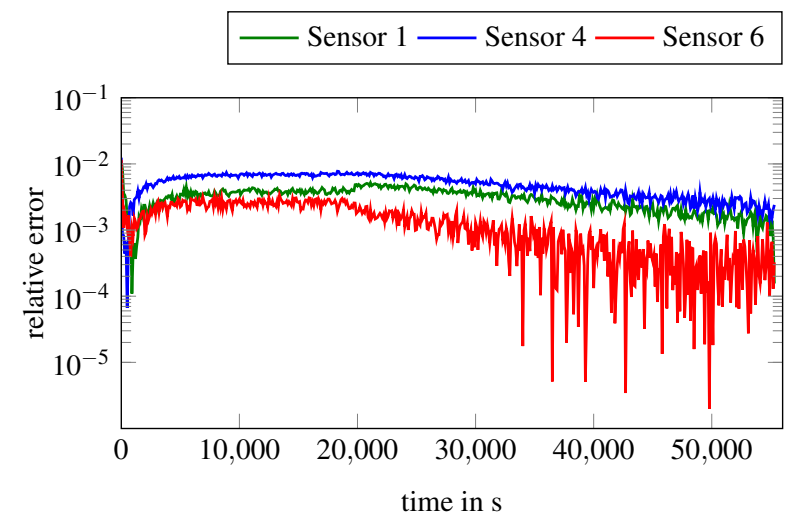

Figure 8: Relative error of the measured outputs $\hat{\mathbf{y}}$ and computed outputs $\mathbf{y}_{r}$ for Sensors 1,4 and 6.

\section{Conclusions}

We have presented an optimal control approach to solve the IHCP of reconstructing a thermal load based on given data measurements. The described ansatz requires the solution of a DRE (9). For large-scale dynamical systems, the usage of low-rank based algorithms is recommended for the solution of the DRE. However, practical applications are often grounded on large time intervals. This directly leads to significant computational times and storage consumption. Therefore, model order reduction is applied to the linear time invariant large-scale system (4), (5). Then the DRE and hence the IHCP is solved based on the small-scale reduced order model (13). The results show that the input signal $\mathbf{u}$ and the associated outputs $\mathbf{y}$ can be reconstructed up to a relative error in the range of one per thousand. The investigation of an LQG design based approach in order to handle the measurement noise more appropriate is postponed to future work.

Anyway, the approach presented above relies on considering a linear model in order to describe the underlying tem- 
perature field. Leaving the admissible ambit of the linear model by e.g., considering non-linear boundary conditions or temperature dependent model parameters, yields in the inadmissibility of the procedure.

\section{Acknowledgments}

This research was funded by the Deutsche Forschungsgemeinschaft (DFG) within the Collaborative Research Center/ Transregio 96 Thermo-Energetic Design of Machine Tools

\section{References}

1. Banks, H.T., Ito, K.: A numerical algorithm for optimal feedback gains in high dimensional linear quadratic regulator problems. SIAM J. Cont. Optim. 29(3), 499-515 (1991)

2. Banks, H.T., Kunisch, K.: The linear regulator problem for parabolic systems. SIAM J. Cont. Optim. 22, 684-698 (1984)

3. Benner, P.: Computational methods for linear-quadratic optimization. Supplemento ai Rendiconti del Circolo Matematico di Palermo, Serie II No. 58, 21-56 (1999)

4. Benner, P., Kürschner, P., Saak, J.: Efficient handling of complex shift parameters in the low-rank Cholesky factor ADI method. Numer. Algorithms 62(2), 225-251 (2013). DOI 10.1007/s11075-012-9569-7

5. Benner, P., Kürschner, P., Saak, J.: Self-generating and efficient shift parameters in ADI methods for large Lyapunov and Sylvester equations. Electron. Trans. Numer. Anal. 43, 142-162 (2014).

6. Benner, P., Li, J.R., Penzl, T.: Numerical solution of large Lyapunov equations, Riccati equations, and linear-quadratic control problems. Numer. Lin. Alg. Appl. 15(9), 755-777 (2008)

7. Benner, P., Mena, H.: Numerical solution of the infinitedimensional LQR-problem and the associated differential Riccati equations. Preprint MPIMD/12-13, MPI Magdeburg Preprint (2012). Available from http://www.mpi-magdeburg.mpg. de/preprints/

8. Benner, P., Mena, H.: Rosenbrock methods for solving Riccati differential equations. IEEE Trans. Automat. Control 58(11), 29502957 (2013)

9. Bonesky, D., Dahlke, S., Maass, P., Raasch, T.: Adaptive wavelets methods and sparsity reconstruction for inverse heat conduction problems. Advances in Computational Mathemathics 33(4), 385411 (2010)

10. Enns, D.F.: Model reduction with balanced realizations: An error bound and a frequency weighted generalization. In: Proc. 23rd IEEE Conf. Decision Contr., vol. 23, pp. 127-132 (1984)

11. Grossmann, C., Roos, H.G., Stynes, M.: Numerical Treatment of Partial Differential Equations. Springer-Verlag Berlin Heidelberg (2007)

12. Hairer, E., Wanner, G.: Solving Ordinary Differential Equations II - Stiff and Algebraic Problems, second edn. Springer, Berlin Heidelberg (2002)

13. Kindermann, S., Navasca, C.: Optimal control as a regularization method for ill-posed problems. Journal of Inverse and Ill-posed Problems 14, 685-703 (2006)

14. Lamm, P.K.: Future-sequential regularization methods for illposed Volterra equations. Journal of Mathematical Analysis and Application pp. 469-494 (1996)

15. Lang, N., Mena, H., Saak, J.: An $L D L^{T}$ factorization based ADI algorithm for solving large scale differential matrix equations. Proc. Appl. Math. Mech. 14(1) (2014)
16. Li, J.R., White, J.: Low rank solution of Lyapunov equations. SIAM J. Matrix Anal. Appl. 24(1), 260-280 (2002)

17. Locatelli, A.: Optimal Control: An Introduction. Birkhäuser, Basel, Switzerland (2001)

18. Louis, A.K.: Inverse und schlecht gestellte Probleme. Teubner (1989)

19. Moore, B.C.: Principal component analysis in linear systems: controllability, observability, and model reduction. IEEE Trans. Automat. Control AC-26(1), 17-32 (1981)

20. Neugebauer, R., Drossel, W.G., Ihlenfeldt, S., Richter, C.: Thermal interactions between the process and workpiece. Procedia CIRP pp. 63-66 (2012). DOI 10.1016/j.procir.2012.10.012

21. Penzl, T.: A cyclic low rank Smith method for large sparse Lyapunov equations. SIAM J. Sci. Comput. 21(4), 1401-1418 (2000)

22. Penzl, T.: Eigenvalue decay bounds for solutions of Lyapunov equations: the symmetric case. Systems Control Lett. 40, 139144 (2000)

23. Penzl, T.: Algorithms for model reduction of large dynamical systems. Linear Algebra Appl. 415(2-3), 322-343 (2006). (Reprint of Technical Report SFB393/99-40, TU Chemnitz, 1999.)

24. Schindler, S., Zimmermann, M., Aurich, J.C., Steinmann, P.: Finite element model to calculate the thermal expansions of the tool and the workpiece in dry turning. Procedia CIRP 14, 535-540 (2014)

25. Schindler, S., Zimmermann, M., Aurich, J.C., Steinmann, P.: Thermo-elastic deformations of the workpiece when dry turning aluminum alloys - a finite element model to predict thermal effects in the workpiece. CIRP Journal of Manufacturing Science and Technology 7, 233-245 (2014). DOI 10.1016/j.cirpj.2014.04.006

26. Schweinoch, M., Joliet, R., Kersting, P.: Predicting thermal loading in nc milling processes. Production Engineering (2014). DOI 10.1007/s11740-014-0598-z

27. Strauss, W.A.: Partial Differential Equations: An Introduction. Whiley \& Sons Ltd, Brown University (2008) 